\title{
Inpatient Dermatology Consultations in a General Surgery Ward in a Tertiary Hospital in China: A Retrospective Study of 251 Patients
}

\author{
Hanlin Zhang · Keyun Tang • Rouyu Fang · Hongzhong Jin • \\ Qiuning Sun (1)
}

Received: February 11, 2021 / Published online: April 22, 2021

(c) The Author(s) 2021

\begin{abstract}
Introduction: Dermatologists play essential roles in providing dermatology consultations to inpatients admitted to hospital for care in another speciality ward. Data on dermatology consultations provided to inpatients admitted to general surgery wards are limited. The aim of this study was to analyze the reasons for and diagnoses of consultations provided by dermatologists to hospitalized patients in a general surgery ward and compare the provisional diagnoses by surgical residents and the final diagnoses by dermatologists.

Methods: Electronic health records were retrieved for patients admitted to a general surgery ward who received dermatology consultations while inpatients in Peking Union Medical College Hospital between 1 September 2015 and 31 August 2020. Sex, age, surgical diagnosis, reason for dermatology consultation,
\end{abstract}

Hanlin Zhang and Keyun Tang contributed equally to this study.

H. Zhang $\cdot$ K. Tang $\cdot$ R. Fang $\cdot$ H. Jin $(\varangle)$.

Q. Sun $(\bowtie)$

Department of Dermatology, Peking Union Medical

College Hospital, No.1, Shuaifuyuan, Dongcheng

District, Beijing, China

e-mail: jinhongzhong@263.net

Q. Sun

e-mail: doctorjenny1@163.com provisional diagnosis by surgical residents, and final diagnosis by dermatologists were reviewed. Results: A total of 262 dermatology consultations for 251 patients $(n=251,123$ women and 128 men) were identified, of whom $240(95.6 \%)$ required only one consultation and 11 (4.4\%) required two. Dermatology consultations were classified into three categories: preoperative consultation $(n=45,17.9 \%)$, postoperative consultation ( $n=65,25.9 \%)$, and consultation unrelated to general surgery diseases or treatments (including surgery) ( $n=141,56.2 \%)$. For consultations falling in the category preoperative consultation, common reasons for the consultation were: to evaluate whether the current treatment plan for previously diagnosed skin diseases needed to be changed; to evaluate and manage skin problems that emerged after admission; to evaluate syphilis; and to evaluate whether previously diagnosed skin diseases would affect surgical incision or wound healing. Drug eruption, dermatitis/eczema, infectious skin disorders, and urticaria were the most common skin diseases in the hospitalized general surgery patients. Only 32 (12.7\%) provisional diagnoses were made, of which 25 (78.1\%) were correct and seven (21.9\%) were incorrect. Surgical residents mainly had difficulty distinguishing herpes zoster, drug eruption, and infectious skin disorders from dermatitis/eczema.

Conclusion: Our results facilitate the understanding of inpatient dermatology 
consultations in general surgery wards and may help in the design of future educational materials and/or management guidelines.

Keywords: Inpatient dermatology consultation; Consultative dermatology; General surgery; Resident training

\section{Key Summary Points}

Dermatologists play essential roles in providing dermatology consultations to inpatients hospitalized for care in another speciality discipline.

This study aims to analyze the reasons for and diagnoses from consultations provided by dermatologists to hospitalized patients in a general surgery ward, and to compare provisional diagnoses by surgical residents and final diagnoses by dermatologists.

Dermatology consultations were classified into three categories: preoperative consultation (17.9\%), postoperative consultation $(25.9 \%)$, and consultation unrelated to general surgery diseases or treatments (including surgery) (56.2\%).

Drug eruption, dermatitis/eczema, infectious skin disorders, and urticaria were common skin diseases in hospitalized general surgery patients.

Our results facilitate the understanding of inpatient dermatology consultations in general surgery wards and may help in the design of future educational materials and/or management guidelines.

\section{DIGITAL FEATURES}

This article is published with digital features, including a summary slide, to facilitate understanding of the article. To view digital features for this article go to https://doi.org/10.6084/ m9.figshare.14340272.

\section{INTRODUCTION}

Dermatology is primarily an outpatient-based service; however, dermatologists also play essential roles in providing dermatology consultations to inpatients hospitalized for care in other speciality disciplines $[1,2]$. Timely and accurate dermatology consultations can benefit the hospitalized patients in terms of diagnosis and treatment of skin diseases [3, 4]. A retrospective study of 731 referrals for dermatology consultations showed that there was not always a good correspondence between the provisional diagnoses made by the referring doctors and the final diagnoses confirmed by dermatologists, with $30.2 \%$ correct diagnoses, $35.2 \%$ incorrect diagnoses, and $34.6 \%$ no provisional diagnoses [5]. Another study also showed that dermatology consultations were associated with a reduction in the adjusted hospital length of stay by 2.64 days [6]. Inpatient dermatology consultation can shorten hospitalizations and reduce costs [7]. Retrospective analysis of inpatient dermatology consultations also facilitates dermatology education among specialists in other disciplines by increasing the latter's awareness of skin diseases [8]. The collaboration between dermatologists and physicians in other disciplines greatly relies on consultations and improved quality of patient care [2].

The spectrum of skin diseases in patients admitted to non-dermatology hospital wards has been reviewed and analyzed, including for internal medicine [9], hematology [10], neurosurgery [2], and obstetrics and gynecology [11]. However, data on dermatology consultations for inpatients admitted to general surgery wards are limited. The aim of this study was to analyze the reasons for and diagnoses from consultations provided by dermatologists to hospitalized patients in a general surgery ward. Provisional diagnoses by surgical residents and final diagnoses by dermatologists were also compared. Knowledge of these reasons and diagnoses may help dermatologists provide better care for hospitalized patients in surgical wards. This 
study might also facilitate dermatology education among future surgeons.

\section{METHODS}

Electronic health records were retrieved for patients admitted to a general surgery ward who received dermatology consultations while an inpatient of Peking Union Medical College Hospital, a tertiary hospital in Beijing, China, over a 5-year period between 1 September 2015 and 31 August 2020. Inpatient dermatology consultations were requested by physicians from other departments and conducted by senior residents or junior attending dermatologists.

Sex, age, surgical diagnosis, reason for dermatology consultation, provisional diagnosis by surgical residents, and final diagnosis by dermatologists were reviewed. The diagnoses of skin diseases were made according to the International Classification of Disease, 10th Revision. Diseases were classified by two of the authors (HZ and KT) according to previously published articles on inpatient dermatology consultations $[2,4,10,12]$, and the classification was re-checked by another two authors (HJ and QS, both professors in dermatology). This study was approved by the Institutional Review Board at Peking Union Medical College Hospital (Number: S-K1589).

\section{RESULTS}

\section{Baseline Characteristics}

A total of 262 consultations for 251 patients ( $n=251,123$ women, 128 men) were identified and reviewed. The average ( \pm standard deviation [SD]) age of the patients attending a consultation was $54.23 \pm 16.24$ (median 56) years. Of these 251 patients, $240(95.6 \%)$ required only one consultation and 11 patients $(4.4 \%)$ required two. Patients who needed general surgery were admitted to the general surgery ward for pancreas diseases $(n=51)$, stomach diseases $(n=38)$, thyroid diseases $(n=33)$, rectal diseases $(n=32)$, colon diseases $(n=24)$, gallbladder diseases $(n=13)$, bile duct diseases $(n=11)$, and others $(n=49)$.

\section{Reason for Dermatology Consultations and Spectrum of Skin Diseases}

Dermatology consultations (evaluation and management of dermatologic conditions) were classified into three categories according to electronic health records: preoperative consultation related to general surgery diseases or treatments (including surgery) $(n=45,17.9 \%)$; postoperative consultation related to general surgery diseases or treatments (including surgery) ( $n=65,25.9 \%)$; and consultation unrelated to general surgery diseases or treatments (including surgery) ( $n=141,56.2 \%)$.

Table 1 shows the reasons for the dermatology consultations and the final diagnoses by dermatologists for preoperative consultations related to general surgery diseases or treatments (including surgery) $(n=45)$. The most common reason for a consultation was to evaluate whether the current treatment plan for a previously diagnosed skin disease needed to be changed before surgery $(n=14)$. Other reasons included the need to evaluate and manage skin problems that emerged after admission $(n=11)$; assess syphilis (due to positive rapid plasma reagin [RPR] test and/or positive Treponema pallidum particle agglutination assay [TPPA] $(n=10)$; evaluate whether previously diagnosed skin diseases would affect surgical incision or wound healing $(n=5)$; evaluate whether previously diagnosed skin diseases would affect the feasibility of surgery $(n=3)$; and assess whether current skin diseases were related to general surgery diseases and assist in treatment $(n=2)$.

Tables 2 and 3 show the spectrum of skin diseases that were the reasons for postoperative consultations related to general surgery diseases or treatments (including surgery) $(n=65)$, and consultations unrelated to general surgery diseases or treatments $(n=141)$. Reasons for consultations under these circumstances are all about asking for a diagnosis and treatment for skin lesions and treatment for previously diagnosed skin diseases. The most common reason for postoperative consultations was drug 
Table 1 Reasons for dermatology consultations and final diagnoses by dermatologists for preoperative consultations related to general surgery diseases or treatments (including surgery)

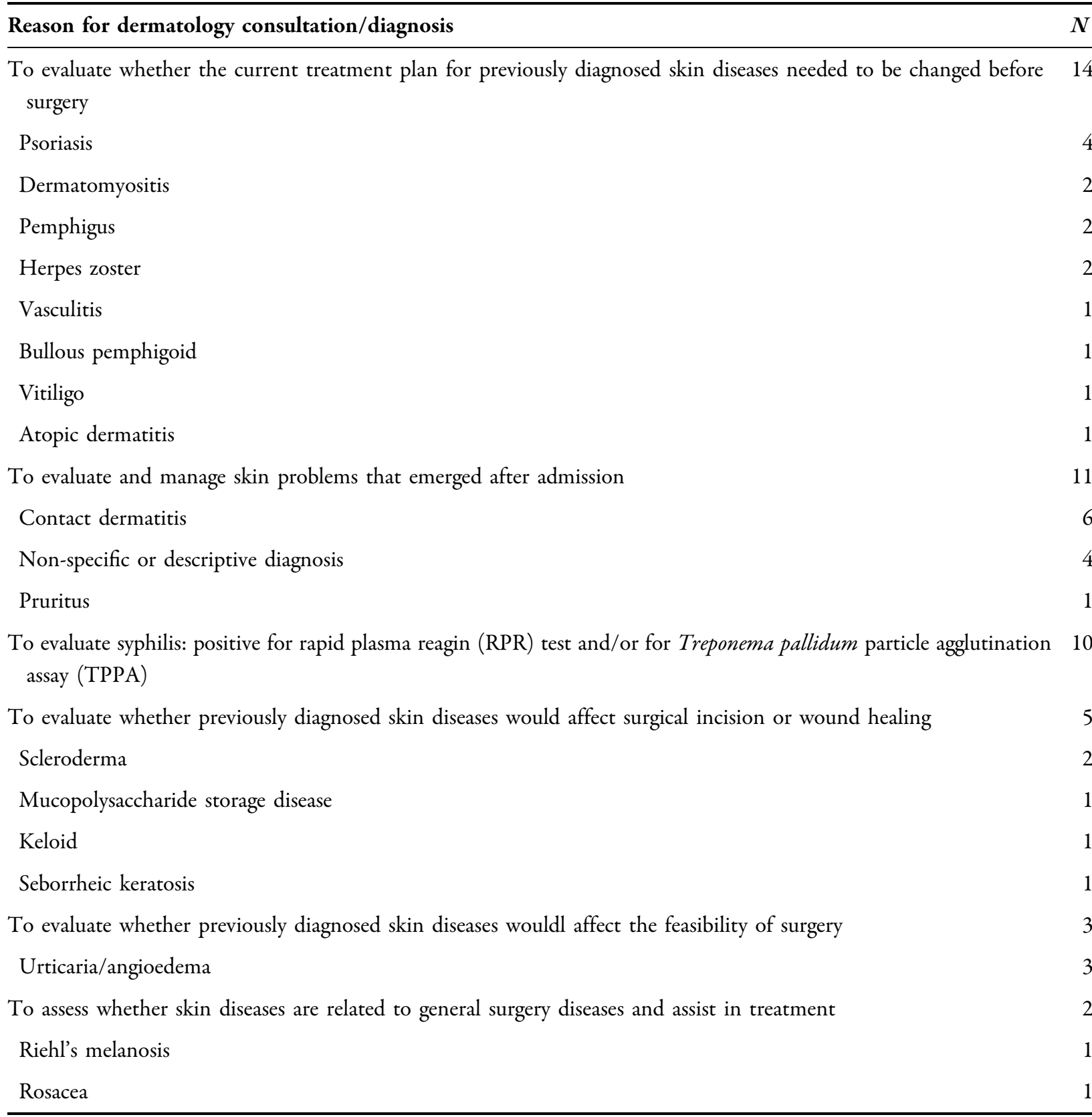

eruption $(n=22)$, followed by dermatitis and eczema $(n=21)$, infectious skin disorders $(n=16)$, non-specific or descriptive diagnosis $(n=5)$, and acne fulminans $(n=1)$. The most common reason for consultations unrelated to general surgery diseases or treatments was dermatitis and eczema $(n=67)$, followed by infectious skin disorders $(n=20)$, urticaria $(n=14)$, non-specific or descriptive diagnosis $(n=5)$, and other diseases ( $n=35$; for psoriasis, $n=6$; for each of the other specific diseases, $n<5$ ).

\section{Provisional Diagnoses and Final Diagnoses}

In the context of the consultation system, surgical residents, in addition to recording the 
Table 2 Skin diseases as the reason for postoperative consultations related to general surgery diseases or treatments (including surgery)

\begin{tabular}{lr}
\hline Diagnosis & $\boldsymbol{N}$ \\
\hline Drug eruption & 22 \\
Dermatitis/eczema & 21 \\
Contact dermatitis & 19 \\
Other types of dermatitis or eczema & 2 \\
Infectious skin disorders & 16 \\
Herpes zoster & 8 \\
Bacterial infections & 6 \\
Herpes simplex & 1 \\
Candidiasis & 1 \\
Non-specific or descriptive diagnosis & 5 \\
Acne fulminans & 1 \\
\hline
\end{tabular}

reasons for a dermatology consultation, medical history and current condition of the patient, and description of skin lesions, can also write and submit provisional diagnoses, which will be confirmed or revised by dermatologists. In this study, only 32 provisional diagnoses were provided, of which 25 (78.1\%) were correct (diagnoses by surgical residents and dermatologists were the same) and seven (21.9\%) were incorrect (diagnoses by surgical residents and dermatologists differed). The seven incorrect provisional diagnoses and corresponding final diagnoses are listed in Table 4.

\section{DISCUSSION}

In this study, we summarized the reasons for consultations with dermatologists and the diagnoses provided by dermatologists based on these consultations, for hospitalized patients in a general surgery ward in a tertiary hospital in China. Different medical departments and health systems assess the spectrum of skin diseases differently. However, the spectrum assessed in our study is similar to that used in previous studies, with dermatitis/eczema, skin
Table 3 Skin diseases as the reason for consultations unrelated to general surgery diseases or treatments

\begin{tabular}{lc}
\hline Diagnosis & $N$ \\
\hline Dermatitis/eczema & 67
\end{tabular}

Contact dermatitis $\quad 7$

Pruritus 6

Lichen simplex chronicus $\quad 4$

Stasis dermatitis 4

Atopic dermatitis 2

Prurigo simplex 1

Prurigo nodularis 1

Non-specific dermatitis or eczema $\quad 42$

Infectious skin disorders 20

$\begin{array}{ll}\text { Herpes zoster } & 7\end{array}$

Bacterial infections 5

Mycoses 4

Herpes simplex 3

Varicella 1

Urticaria 14

Other diseases $\quad 35$

Psoriasis 6

Rosacea 4

Miliaria 4

Pigmented purpuric dermatosis 4

Decubitus ulcer 3

Benign familial pemphigus 3

Vasculitis 3

Amyloidosis of skin 2

Seborrheic keratosis $\quad 2$

Lipoma 1

Burns 1

Acne 1

Fibroma 1

Non-specific or descriptive diagnosis 5 
Table 4 The seven incorrect provisional diagnoses by surgical residents and corresponding final diagnoses by dermatologists

\begin{tabular}{ll}
\hline $\begin{array}{l}\text { Provisional diagnosis } \\
\text { by surgical residents }\end{array}$ & $\begin{array}{l}\text { Final diagnosis } \\
\text { by dermatologists }\end{array}$ \\
\hline Nevus & Seborrheic keratosis \\
Eczema & Mycosis \\
Dermatitis & Drug eruption \\
Dermatitis & Herpes zoster \\
Acne & Rosacea \\
Dermatitis & Bacterial infection \\
Drug eruption & Eczema, non-specific \\
\hline
\end{tabular}

infections, and drug eruptions accounting for most dermatologic diagnoses [5, 13]. We also summarized seven misdiagnoses from surgical residents, and the corresponding final diagnoses from dermatologists.

Motivations for preoperative consultations were summarized in detail in the medical records. It is important that dermatologists take responsibility to evaluate whether the current treatment plan for previously diagnosed skin diseases, such as psoriasis, dermatomyositis, pemphigus, vasculitis, bullous pemphigoid, vitiligo, and atopic dermatitis, needs to be modified before surgery. Dermatologists emphasize the need for a long follow-up period for inflammatory or autoimmune diseases, to better control and prevent relapses [14-16]. Two patients in our study previously diagnosed with herpes zoster during the recovery periods also asked for dermatology consultations on drug adjustment. Herpes zoster should be given more attention, especially when caring for patients who are immunocompromised or have chronic disorders [17]. Dermatologists also play a role in evaluating and providing suggestions on syphilis, which could ensure the medical safety of syphilis patients and medical staff. Surgeons also asked for evaluations regarding whether previously diagnosed skin diseases, such as scleroderma, keloid, and seborrheic keratosis, would affect surgical incision or wound healing. Dermatologic evaluation is also recommended for patients with urticaria/angioedema, to ensure that the disease is well controlled. Severe urticaria/angioedema may endanger the lives of patients and if present, treatment and/or cancellation/postponement of surgery would be required.

Contact dermatitis was common among the patients included in our study, although most diagnoses of dermatitis and eczema were not specific. Sweating, detergents, soaps, antiseptics, and occlusive dressings for wounds may all contribute to contact dermatitis [2, 18]. Stasis dermatitis should also be treated under the guidance of dermatologists, especially in elderly patients and those overweight or obese and those have to stand for a long time for their work [19]. Diagnosis and management of drug eruption are essential tasks for dermatologists. In our study population, drug eruption accounted for $33.8 \%$ of all postoperative consultations related to general surgery diseases or treatments, and was present in $8.8 \%$ of the 251 patients. Early diagnosis and treatment are important for patients with drug eruption, especially severe drug eruption [20]. Hung and colleagues reviewed drug allergies in a surgical population, and concluded that most reported drug allergies were for antibiotics (50\%), opioids (27\%), non-steroidal anti-inflammatory agents (10\%), and sedatives (5\%) [21]. Weinkle et al. [3] summarized the clinical characteristics of their patients with Stevens-Johnson syndrome and toxic epidermal necrolysis and concluded that Nikolsky sign, atypical targets, fever, and lymphopenia could predict the probability of these two diseases.

Of all 251 patients in our study, only 32 $(12.7 \%)$ received provisional diagnoses from surgical residents. Residents should be encouraged to present their provisional diagnoses as this would enable dermatologists to provide guidance. Non-dermatologists may find it difficult to care for hospitalized patients with skin diseases because of the wide range of dermatologic conditions that can present in patients [2]. Falanga et al. [22] reported that dermatologic consultations could result in the dermatologic diagnosis and treatment being changed in more than $60 \%$ of hospitalized patients. Here we summarized the seven incorrect provisional diagnoses provided by surgical residents and 
corresponding final diagnoses by dermatologists. Overall, surgical residents were found to have difficulty distinguishing herpes zoster, drug eruption, and infectious skin disorders from dermatitis/eczema. This result suggests that the diagnosis and treatment of common skin diseases should be popularized in residents who are directly in charge of hospitalized patients.

Inpatient dermatology consultations can help identify skin diseases and skin lesions secondary to other diseases or secondary to treatments, and assist in the diagnosis and treatment of systemic diseases, thereby benefiting patients by providing multidisciplinary care [23, 24]. Such consultations also have educational significance for both dermatologists and non-dermatologists. Inpatient dermatology consultation is essential training for senior residents and junior attendings in departments of dermatology in China. Although some consultations may be difficult, a wide variety of clinical scenarios can enrich their professional knowledge and broaden their horizons [25]. Residents from other departments can also learn dermatologic knowledge from dermatologists by providing a provisional diagnosis and then following specialist management of the case. Inpatient dermatology consultations also help improve the understanding of skin diseases, especially comorbidities and systemic treatments, of senior residents . Among our cases, the surgical resident described a 55-year old gastric cancer patient with "erythema, flushing, and multiple papules on the face", and made a provisional diagnosis of "acne." However, the dermatologist made the final diagnosis of "rosacea." Helicobacter pylori is known to play a role in the development of rosacea, which is also one of the main causes of chronic gastritis, gastric cancer, and gastrointestinal ulcers $[26,27]$. Therefore, such consultations result in providing better patient care and help dermatologists and surgeons better understand rosacea and gastric cancer.

Dermatology consultations, especially teledermatology consultations, havealso played a role in fighting against COVID-19. In West China Hospital, dermatologists have been providing free online and telephone consultations to reduce the flow of patients to the hospital while still meeting their medical needs amid the COVID-19 pandemic [28]. Dermatologists working side by side with healthcare professionals from other departments have helped treat skin diseases associated with COVID-19 $[29,30]$. Teleconsultation can be provided by dermatologists for COVID-19 patients; if necessary, bedside consultation is also provided [31]. Teledermatology consultation can also be used to provide underresourced hospitals with access to dermatologists, as well as to maintain resident education during COVID-19 [32, 33]. To date, inpatient teledermatology has not been applied in our hospital, but this approach might be considered in the future to help meet the demand for inpatient skin disease treatment and the prevention of misdiagnosis, unnecessary costs, and unnecessary treatments [34].

This study has several limitations. Due to the retrospective nature of the study, include figures and detailed descriptions of skin lesions were not available; thus, precisely which drug eruptions and skin infections occurred were hard to identify. Also, this study was conducted only in a single tertiary medical center, and the department of breast surgery and liver surgery are separate from the department of general surgery in this hospital, which may have introduced bias into the study. Larger long-term prospective studies are warranted in the future.

\section{CONCLUSION}

To our knowledge, this is the first long-term study on inpatient dermatology consultations in general surgery wards. In this study, we enrolled 251 patients admitted to a general surgery ward in a hospital in China and analyzed the reasons for and diagnoses from inpatient consultations with dermatologists. Dermatology consultations could be classified into three categories: preoperative consultation $(n=45,17.9 \%)$; postoperative consultation $(n=65,25.9 \%)$; and consultation unrelated to general surgery diseases or treatments (including surgery) ( $n=141,56.2 \%)$. For consultations falling in the category preoperative consultation, commons reasons for the consultation 
were: to evaluate whether the current treatment plan for previously diagnosed skin diseases needed to be changed; to evaluate and manage skin problems that emerged after admission; to evaluate syphilis; and to evaluate whether previously diagnosed skin diseases would affect surgical incision or wound healing. Drug eruption, dermatitis/eczema, infectious skin disorders, and urticaria are common skin diseases in hospitalized patients in the general surgery ward. Only $32(12.7 \%)$ provisional diagnoses were provided, of which $25(78.1 \%)$ were correct and seven $(21.9 \%)$ were incorrect. Our results facilitate the understanding of inpatient dermatology consultations in general surgery wards and may help in the design of future educational materials and/or management guidelines. Inpatient dermatology consultations benefit the hospitalized patient in terms of the diagnosis and treatment of skin diseases and provide a means to educate both dermatologists and non-dermatologists.

\section{ACKNOWLEDGEMENTS}

Funding. No sponsorship was received for this study or publication of this article. The Rapid Service Fee was funded by Peking Union Medical College Hospital.

Authorship. All named authors meet the International Committee of Medical Journal Editors (ICMJE) criteria for authorship for this article, take responsibility for the integrity of the work as a whole, and have given their approval for this version to be published.

Authorship Contributions. Hanlin Zhang and Keyun Tang designed the study and prepared the manuscript. Rouyu Fang made the tables. Hongzhong Jin and Qiuning Sun supervised the study and revised the manuscript.

Disclosures. Hanlin Zhang, Keyun Tang, Rouyu Fang, Hongzhong Jin, Qiuning Sun declare that they have nothing to disclose.
Compliance with Ethics Guidelines. This study was approved by the Institutional Review Board at Peking Union Medical College Hospital.

Data Availability. The datasets generated during and/or analyzed during the current study are available from the corresponding author on reasonable request.

Open Access. This article is licensed under a Creative Commons Attribution-NonCommercial 4.0 International License, which permits any non-commercial use, sharing, adaptation, distribution and reproduction in any medium or format, as long as you give appropriate credit to the original author(s) and the source, provide a link to the Creative Commons licence, and indicate if changes were made. The images or other third party material in this article are included in the article's Creative Commons licence, unless indicated otherwise in a credit line to the material. If material is not included in the article's Creative Commons licence and your intended use is not permitted by statutory regulation or exceeds the permitted use, you will need to obtain permission directly from the copyright holder. To view a copy of this licence, visit http://creativecommons.org/licenses/by$\mathrm{nc} / 4.0 /$.

\section{REFERENCES}

1. Uzuncakmak TK, Bayazit S, Askin O, Engin B, Kutlubay $\mathrm{Z}$. Inpatient dermatology consultations during COVID 19 pandemic in a tertiary referral center. Dermatol Ther. 2020;33(6):e13883. https://doi.org/ 10.1111/dth.13883.

2. Kim KM, Kim HS, Yu J, Kim JT, Cho SH. Analysis of dermatologic diseases in neurosurgical in-patients: a retrospective study of 463 cases. Ann Dermatol. 2016;28(3):314-20. https://doi.org/10.5021/ad. 2016.28.3.314.

3. Weinkle A, Pettit C, Jani A, Keller J, Lu Y, Malachowski S, et al. Distinguishing Stevens-Johnson syndrome/toxic epidermal necrolysis from clinical mimickers during inpatient dermatologic consultation-a retrospective chart review. J Am Acad Dermatol. 2019;81(3):749-57. https://doi.org/10. 1016/j.jaad.2019.05.061. 
4. Fayne R, Castillo DE, Sanchez N, et al. Dermatology consultation service at a large metropolitan hospital system serving minority populations. J Eur Acad Dermatol Venereol. 2020;34(9):2120-6. https://doi. org/10.1111/jdv.16565.

5. Tay LK, Lee HY, Thirumoorthy T, Pang SM. Dermatology referrals in an East Asian tertiary hospital: a need for inpatient medical dermatology. Clin Exp Dermatol. 2011;36(2):129-34. https://doi.org/10. 1111/j.1365-2230.2010.03923.x.

6. Milani-Nejad N, Zhang M, Kaffenberger BH. Association of dermatology consultations with patient care outcomes in hospitalized patients with inflammatory skin diseases. JAMA Dermatol. 2017;153(6):523-8. https://doi.org/10.1001/ jamadermatol.2016.6130.

7. Zhong CS, Mostaghimi A, Kroshinsky D, Nambudiri VE. Revenue generation of dermatology inpatient consultations: a retrospective multi-institutional evaluation of academic hospital-based consults. J Am Acad Dermatol. 2020. https://doi.org/10. 1016/j.jaad.2020.08.044.

8. Tsai S, Ezaldein HH, Conic RRZ, Merati M, Scott JF. Inpatient dermatology consultations and the July effect: a retrospective cohort study. J Am Acad Dermatol. 2019;81(1):267-9. https://doi.org/10. 1016/j.jaad.2019.02.030.

9. Antic M, Conen D, Itin PH. Teaching effects of dermatological consultations on nondermatologists in the field of internal medicine. A study of 1290 inpatients. Dermatology. 2004;208(1):32-7. https://doi.org/10.1159/000075043.

10. Alasmari AA, Hakeem AH, Bin Saleh FS, Alsaigh SY, Al Ajroush W, Layqah LA, et al. Pattern of dermatological disease encountered in a hematology ward: a retrospective analysis of dermatology consultation in a hematology ward in a tertiary care center in Saudi Arabia. Dermatol Res Pract. 2019;2019:9891270. https://doi.org/10.1155/2019/ 9891270 .

11. Noh TK, Haw S, Won $\mathrm{CH}$, et al. A statistical analysis and clinical evaluation of dermatologic consultations in obstetrics and gynecologic inpatients. Korean J Dermatol. 2012;50(11):951-8.

12. Rubegni P, Cevenini G, Lamberti A, et al. Dermatological conditions presenting at the Emergency Department in Siena University Hospital from 2006 to 2011. J Eur Acad Dermatol Venereol. 2015;29(1): 164-8. https://doi.org/10.1111/jdv.12513.

13. Tham WY, Koh HY, Lee HY. Spectrum of dermatological conditions seen in the intensive care unit. Clin Exp Dermatol. 2016;41(8):920-1. https://doi. org/10.1111/ced.12901.
14. Zhao Y, Wang G, Ni W, et al. Visit adherence of mild to moderate psoriasis patients: a mobile-based randomized study. Patient Prefer Adherence. 2020;14:2551-7. https://doi.org/10.2147/ppa. s277103.

15. Fujisawa $\mathrm{T}$, Hozumi $\mathrm{H}$, Kono $\mathrm{M}$, et al. Predictive factors for long-term outcome in polymyositis/dermatomyositis-associated interstitial lung diseases. Respir Investig. 2017;55(2):130-7. https://doi.org/ 10.1016/j.resinv.2016.09.006.

16. Sharma VK, Gupta V, Bhari N, Singh V. Rituximab as an adjuvant therapy for pemphigus: experience in 61 patients from a single center with long-term follow-up. Int J Dermatol. 2019. https://doi.org/10. 1111/ijd.14546.

17. Imafuku S, Matsuki T, Mizukami A, et al. Burden of herpes zoster in the japanese population with immunocompromised/chronic disease conditions: results from a cohort study claims database from 2005-2014. Dermatol Therapy. 2019;9(1):117-33. https://doi.org/10.1007/s13555-018-0268-8.

18. Peñate Y, Guillermo N, Melwani P, Martel R, Borrego L. Dermatologists in hospital wards: an 8-year study of dermatology consultations. Dermatology. 2009;219(3):225-31. https://doi.org/10.1159/ 000232390 .

19. Suehiro K, Morikage N, Yamashita O, et al. Risk factors in patients with venous stasis-related skin lesions without major abnormalities on duplex ultrasonography. Ann Vasc Dis. 2016;9(3):201-4. https://doi.org/10.3400/avd.oa.16-00059.

20. Cheraghlou S, Levy LL. Fixed drug eruptions, bullous drug eruptions, and lichenoid drug eruptions. Clin Dermatol. 2020;38(6):679-92. https://doi.org/ 10.1016/j.clindermatol.2020.06.010.

21. Hung OR, Bands C, Laney G, Drover D, Stevens S, MacSween M. Drug allergies in the surgical population. Can J Anaes. 1994;41(12):1149-55. https:// doi.org/10.1007/bf03020652.

22. Falanga V, Schachner LA, Rae V, et al. Dermatologic consultations in the hospital setting. Arch Dermatol. $1994 ; 130(8): 1022-5$.

23. Temiz SA, Dursun R, Daye M, Ataseven A. Evaluation of dermatology consultations in the era of COVID-19. Dermatol Ther. 2020;33(5):e13642. https://doi.org/10.1111/dth.13642.

24. Pereira AR, Porro AM, Seque CA, Pasin VP, Tomimori J. Inpatient dermatology consultations in renal transplant recipients. Actas Dermo-Sifiliogr. 2018;109(10):900-7. https://doi.org/10.1016/j.ad. 2018.07.009. 
25. Chavez-Alvarez S, Herz-Ruelas M, Ocampo-Candiani J, Ayala-Cortes AS, Gomez-Flores M. Dermatology inpatient consultations in Latin America: 3-year experience in our University Hospital setting. Int J Dermatol. 2019;58(10):1172-4. https:// doi.org/10.1111/ijd.14418.

26. Yang X. Relationship between Helicobacter pylori and Rosacea: review and discussion. BMC Infect Dis. 2018;18(1):318. https://doi.org/10.1186/ s12879-018-3232-4.

27. Wang Y, Zhang H, Fang R, Tang K, Sun Q. The top 100 most cited articles in rosacea: a bibliometric analysis. J Eur Acad Dermatol Venereol. 2020;34(10):2177-82. https://doi.org/10.1111/jdv. 16305 .

28. Chen Y, Pradhan S, Xue S. What are we doing in the dermatology outpatient department amidst the raging of the 2019 novel coronavirus? J Am Acad Dermatol. 2020;82(4):1034. https://doi.org/10. 1016/j.jaad.2020.02.030.

29. Wang Y, Fang R, Zhang H, Tang K, Sun Q. Contributions of dermatologists to COVID-19 research: a brief systematic review. Dermatol Ther. 2020;33(4): e13713. https://doi.org/10.1111/dth.13713.
30. Tang K, Wang Y, Zhang H, Zheng Q, Fang R, Sun Q. Cutaneous manifestations of the Coronavirus Disease 2019 (COVID-19): a brief review. Dermatol Ther. 2020;33(4):e13528. https://doi.org/10.1111/ dth.13528.

31. Zhang H, Tang K, Fang R, Sun Q. What dermatologists could do to cope with the novel coronavirus (SARS-CoV-2): a dermatologist's perspective from China. J Eur Acad Dermatol Venereol. 2020;34(5): e211-2. https://doi.org/10.1111/jdv.16389.

32. Holmes AN, Chansky PB, Simpson CL. Teledermatology consultation can optimize treatment of cutaneous disease by nondermatologists in underresourced clinics. Telemed J E-health. 2020;26(10): 1284-90. https://doi.org/10.1089/tmj.2019.0217.

33. Hammond MI, Sharma TR, Cooper KD, Beveridge MG. Conducting inpatient dermatology consultations and maintaining resident education in the COVID-19 telemedicine era. J Am Acad Dermatol. 2020;83(4):e317-8. https://doi.org/10.1016/j.jaad. 2020.07.008.

34. Georgesen C, Karim SA, Liu R, Moorhead A, Falo LD Jr, English JC 3rd. Inpatient eDermatology (teledermatology) can help meet the demand for inpatient skin disease. Telemed J E-health. 2020;26(7): 872-8. https://doi.org/10.1089/tmj.2019.0147. 